\title{
Thermal lensing characterization of a high-radiance 946 nm planar waveguide laser
}

\author{
S. P. Ng and J. I. Mackenzie \\ ${ }^{1}$ Optoelectronics Research Centre, University of Southampton, Southampton SO17 1BJ, United \\ Kingdom. \\ jim@orc.soton.ac.uk
}

\begin{abstract}
:
We present the characterization of the in-plane thermal lens in a quasi-four-level Nd:YAG planar waveguide (PW) laser configured for high-radiance operation with an external stable-cavity. Our approach utilises the measurement of the laser's output irradiance distribution at the near- and far-field positions concurrently in order to obtain the "real time" beam propagation parameter and thus beam quality factor, $\mathrm{M}^{2}$. Coupled with the knowledge of the intra-cavity-thermallens-dependent beam sizes at an intra-cavity beam waist, the power dependent effective thermal lens focal length was characterized. A thermal lens focal length of $>450 \mathrm{~mm}$ was obtained at all incident pump powers up to the maximum level of $87 \mathrm{~W}$. This characterization enabled the build of a $29 \mathrm{~W} 946 \mathrm{~nm}$ PW laser with a record output radiance of $4.3 \mathrm{TWm}^{-2} \mathrm{sr}^{-1}$.
\end{abstract}

Keywords: Diode-pumped; Solid-state lasers; Waveguide-laser; Quasi-four-level; Nd:YAG

\section{INTRODUCTION}

Since the first demonstration of the quasi-four-level Nd:YAG laser at $946 \mathrm{~nm}$ with a bulk crystal in 1987 [1], incremental improvements in laser performance have been reported using different geometries for the gain medium. Primarily these laser "architectures" mitigate the detrimental thermal effects associated with the waste heat deposited in the active medium during the pumping cycle; these include end-capped composite bulk rods [2], thin-disks [3], and thin crystal fibres [4]. In recent years, the planar waveguide (PW) architecture has also been found to be extremely well suited to this weak laser transition [5, 6]; furthermore the key advantages of the PW geometry have enabled its power scaling into the hundred watt regime [7]. This is primarily the result of the high-gains achievable in the waveguide structure and the excellent thermal management properties attributed to its large cooling surface-area-to-volume ratio, nominally 10 times that of a typical thin disk structure. Nonetheless, while the PW provides spatial-mode control in the guided dimension, in the unguided axis the propagating mode is still susceptible to the in-plane temperature profile and subsequent thermal lensing effects.

In this work, we report the characterization of the in-plane thermal lens in an end-pumped $946 \mathrm{~nm}$ Nd:YAG PW, which has enabled the demonstration of an efficient laser with a record radiance of $>4 \mathrm{TWm}^{-2} \mathrm{sr}^{-1}$ and an order of magnitude improvement on the radiance level of the $105 \mathrm{~W}$ result in reference [7], operating at an output power of $29 \mathrm{~W}$ despite being limited by a reduced available pump power. Key to this result was our approach in determining the cavity mode beam-waist dimensions at the output coupler and corresponding beam quality factor $\left(\mathrm{M}^{2}\right)$ of the laser simultaneously. To do this we re-imaged the cavity-mode beam-waist irradiance distribution in the near-field and far-field (i.e. for the latter in the Fourier plane of a lens) onto one CCD camera, from which the laser's beam parameter product was determined directly by comparing the dimensions of two separate beam irradiance profiles. It transpires that this technique for measuring thermal lens was previously reported by Neuenschwander et al. [8]. Nevertheless it appears that the real strength of this diagnostic method has not been fully realised in subsequent years, even though it effectively provides immediate feedback for optimising the radiance (and can provide full spatial characterization) of any laser system in either pulsed or CW modes of operation. 


\section{METHODOLOGY}

\subsection{Laser characterization technique}

There are several approaches used to estimate the thermal lens in end-pumped lasers, such as pump power or cavity stability test [9-11], interferometric methods [12, 13], and probe-beam techniques $[14,15]$. For a laser resonator with a plane end-mirror there must be a beam waist positioned at its surface. It can be assumed that the thermal load in the PW produces a graded transverse temperature distribution in the unguided axis only, characteristic of the 1-dimensional heat flow out of the waveguide core. Using an ABCD matrix model for the laser resonator, the beam waist dimensions at the plane end-mirrors can be predicted by approximating the thermal lens with an in-plane cylindrical lens whose focal length is pump-power dependent. The dioptric power of the intra-cavity thermal lens can be determined as a fitted parameter in a cavity model by measuring the output beam characteristics as it propagates away from the resonator beam waist, i.e. from a plane output-coupling mirror for example, once the beam waist size and $\mathrm{M}^{2}$ parameter are identified.

Employing re-imaging techniques, the beam radii at a plane output-coupling mirror can be determined for any given cavity arrangement and thermal-lens condition. Moreover with a separate optical arrangement the laser's far-field irradiance distribution at the Fourier (focal) plane of a lens can be measured simultaneously. The combination of these two measurements gives the beam parameter product of the output. Noting the developments in computers, beam profiling software, and CCD cameras in recent years it is now possible to measure multiple beams at once with one device in almost real time. In addition, the collocated far-field and near-field image planes in our arrangement enabled us to optimise the beam size on the detector for both the near- and far-fields through the selection of the focal lengths $f_{1}-f_{3}$ (whose relationship is explained below), and takes into account the resolution limits of the beam profiler system. This approach not only gives an explicit measurement of the beam-waist radii at the exit of the laser cavity, but also the divergence characteristics, and hence, $\mathrm{M}^{2}$ beam propagation parameter.

To appreciate the simplicity of this approach, it is enlightening to consider the underlying geometric equations. Firstly we start with a multimode beam waist, $W_{0}$, given by [16]:

$$
W_{0}=M \omega_{0}
$$

where $\omega_{0}$ is the beam waist radii of the embedded fundamental mode, and $M$ is the square-root of the beam propagation parameter. The cavity mode beam-waist is fixed in position at the plane end-mirror of the linear cavity and can be traced back through the cavity to the other end-mirror or forwards out of the resonator. A simple imaging telescope arrangement will produce a magnified image, $\mathrm{W}_{\mathrm{nf}}$, defined by the ratio of the focal lengths of the constituent lenses $f_{2}$ and $f_{1}$, i.e. [17]:

$$
W_{n f}=\frac{f_{2}}{f_{1}} W_{0}
$$

It is important to note that this condition is independent of the beam propagation parameter.

Alternatively the irradiance distribution measured at the focal plane of a lens, $f_{3}$, is the Fourier transform of the input beam, where the angular component of the input is transferred into a displacement from the optical axis described by [17]:

$$
W_{f f}=\theta_{0} f_{3}
$$

Here $W_{f f}$ is the far-field beam radius for an input beam divergence of $\theta_{0}$. According to Gaussian beam propagation theory, the beam divergence is directly proportional to its wavelength and the beam waist from which it is propagating, [17], and thus:

$$
W_{f f}=\frac{M^{2} \lambda}{\pi W_{0}} f_{3}
$$

Taking the ratio of (4) and (2) we arrive at the expression for the beam propagation factor, dependent only upon measurable quantities,

$$
M^{2}=\frac{\pi}{\lambda} \frac{f_{1}}{f_{2} f_{3}} W_{n f} W_{f f}
$$




\subsection{Experimental setup and measurement}

Radiance scaling of the $946 \mathrm{~nm}$ PW laser was obtained using an extended stable cavity configuration with two different designs for each of the orthogonal axes with respect to the waveguide. As such a z-fold telescope cavity was arranged to achieve a large cavity mode $(\sim 500 \mu \mathrm{m})$ in-the-plane (unguided axis), whereas, an imaging method was used to couple the extended-cavity mode with that of the fundamental waveguide mode at the intra-cavity facet of the waveguide. This design is advantageous for mode matching between the broad "low-radiance" pump beam and resonator mode in the plane, which unfortunately, is affected by even weak aberrations of the near plane-wavefronts characteristic of the large mode size.

The waveguide used in this experiment was a double-clad YAG/ polycrystalline Spinel $\left(\mathrm{MgO}: \mathrm{Al}_{2} \mathrm{O}_{3}\right)$ structure fabricated by Onyx Optics Inc. This direct-bonded composite consists of a $30 \mu \mathrm{m}$ thick lightly doped $\left(0.6\right.$ at. $\left.\% \mathrm{Nd}^{3+}\right) \mathrm{Nd}$ :YAG core layer, two $\sim 21 \mu \mathrm{m}$ thick undoped YAG inner claddings, and two $2.5 \mathrm{~mm}$ thick Spinel outer claddings. The small index step $\left(\Delta \mathrm{n} \approx 2.5 \times 10^{-4}\right)$ between core and inner-YAG-cladding forms a numerical aperture (NA) $\sim 0.03$, creating a slightly multimode waveguide supporting 3 core modes at the $946 \mathrm{~nm}$ lasing wavelength. The index contrast between Spinel $(\mathrm{n} \approx 1.72)$ and YAG $(\mathrm{n} \approx 1.81)$ gives a NA $>0.5$ for the outer cladding where it can theoretically support nearly 90 modes at the pump wavelength of $808 \mathrm{~nm}$. The whole composite structure whose outer dimensions are $5 \times 5 \times 20$ $\mathrm{mm}^{3}$ was clamped between two water-cooled copper blocks that were maintained at $20{ }^{\circ} \mathrm{C}$ during laser operation.

In the experiment, pump light from a custom built combination of six $808 \mathrm{~nm}$ laser-diode bars (details of which can be found in [10]), was focused to a beam radius of $34 \mu \mathrm{m}$ with an $\mathrm{M}_{\mathrm{y}}{ }^{2} \approx 30$ in the guided-axis, and end-coupled into the YAG/ Nd:YAG region, while in the unguided-axis the beam waist, radius $530 \mu \mathrm{m}$ and $\mathrm{M}_{\mathrm{x}}{ }^{2} \approx 270$, was positioned $\sim 11 \mathrm{~mm}$ inside the waveguide. The laser resonator, illustrated in Figure 1, comprised a $\mathrm{z}$-fold configuration (in the same plane as the unguided axis of the waveguide) formed by two highly reflective (HR) concave mirrors $\left(\mathrm{R}_{2}, \mathrm{R}_{3}\right)$, with radii of curvatures $-300 \mathrm{~mm}$ and $-100 \mathrm{~mm}$ respectively, and initially separated by the sum of their focal lengths. The pump input-coupling plane HR mirror was positioned in close proximity $(<100 \mu \mathrm{m})$ to the front facet of the waveguide. A plane partially reflective output-coupling mirror was positioned $55 \mathrm{~mm}$ after $\mathrm{R}_{3}$.

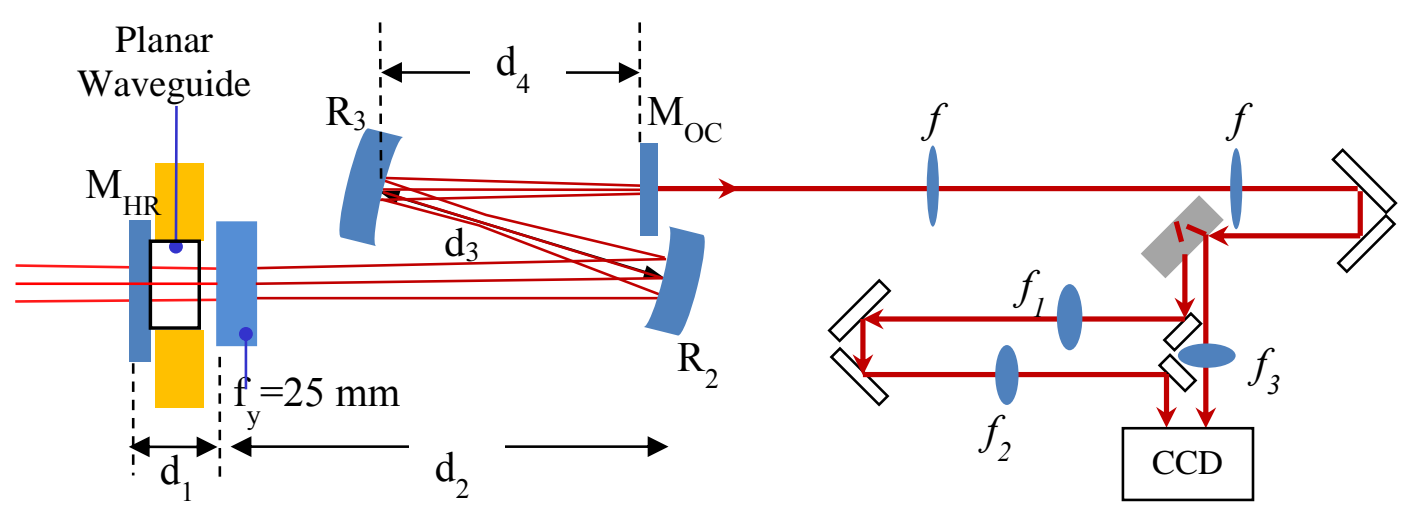

Figure 1: Laser experimental setup and characterization arrangement.

We then built a simple arrangement to simultaneously observe the near- and far-field irradiance distributions of the laser as discussed above. For expedience we re-imaged the output-coupling mirror surface and hence the cavity beam waist with two identical $\mathrm{f}=400 \mathrm{~mm}$ lenses, forming the input plane of our characterization system. In the process we attenuated the beam to a reasonable level for the CCD camera using uncoated glass wedges. As shown in Figure 1, the re-imaged beam was then split into two separate paths, via a tilted thick uncoated plane-parallel optic. One path was focused by an achromatic doublet $f_{3}=100 \mathrm{~mm}$ to its Fourier plane for far field characterization, whereas, the other was magnified by a $2 \times$ afocal telescope $\left(f_{1}=150 \mathrm{~mm}\right.$ and $\left.f_{2}=300 \mathrm{~mm}\right)$, with the first lens $f_{1}$ positioned at its focal length from the input reference plane, thus forming a $2 \times$ magnified near-field image of the output-coupling mirror beam-waist at the output reference (detection) plane. A silicon CCD camera (Spiricon, FireWire) was placed at the detection plane for capturing both near and far field beam irradiance distributions simultaneously, from which the calculated second moments beam dimensions were recorded. The laser's beam quality and fundamental beam waist dimension were then calculated separately for comparison with our modelling. 


\section{RESULTS AND DISCUSSION}

To obtain a rough estimate of the in-plane thermal lens strength we first constructed a simple linear cavity with two plane mirrors and an intra-cavity waveguide coupling cylindrical lens. A HR input-coupling mirror was positioned in close proximity to the waveguide, while the distance between the waveguide and output-coupling mirror was extended until the cavity became unstable that gave an estimated thermal focal length on the order of half a metre. From there we configured the z-fold cavity to be relatively immune to a thermal-lens focal length down to $400 \mathrm{~mm}$, below which the cavity would become unstable. This entailed selecting the separation of the cavity elements to provide an in-the-plane resonator mode size inside the waveguide that was relatively constant with respect to the power of the thermal lens. With reference to Figure 1, a $f_{\mathrm{y}}=25 \mathrm{~mm}$ cylindrical lens with a working distance of $20.4 \mathrm{~mm}$ (at the design wavelength of 587 $\mathrm{nm}$ ) was positioned at $22.9 \mathrm{~mm}\left(\mathrm{~d}_{1}\right)$ from the PW facet, coupling the cavity and waveguide modes. The first folding mirror $R_{2}$ was positioned at $429 \mathrm{~mm}\left(\mathrm{~d}_{2}\right)$ after the cylindrical lens, with a distance of $\sim 200 \mathrm{~mm}\left(\mathrm{~d}_{3}\right)$ to $R_{3}$, and from which there was a $55 \mathrm{~mm}\left(\mathrm{~d}_{4}\right)$ gap to the output-coupling mirror. Typically for this type of cavity configuration, $d_{1}$ is critical for efficient coupling of waveguide and cavity modes, while $d_{3}$ and $d_{4}$ are the major contributors to the in-plane beam size on the in- and output-coupling mirrors.

Monitoring the beam radii in-the-plane $(\mathrm{x})$ and waveguide $(\mathrm{y})$ directions at the output-coupling mirror $\left(\mathrm{W}_{\mathrm{o} / \mathrm{c}}\right)$ as a function of $\mathrm{d}_{1}$ (Figure 2), for a moderate pumping power of $5.5 \mathrm{~W}$, we observe an excellent correlation between the experiment and simulation with an effective thermal lens focal length of $2.3 \mathrm{~m}$. As expected, varying the position of the cylindrical lens had no effect on the in-plane beam size at the output coupler, whereas in the waveguide direction there is a relatively strong dependence. Furthermore it is important to note that for efficient coupling between waveguide and cavity modes it is necessary to match their dimensions at the PW facet. The calculated cavity mode radius $\left(\mathrm{w}_{\mathrm{y}}{ }^{\mathrm{WG}}\right)$ at the facet of the PW as a function of $d_{1}$ is also shown in Figure 2 (dashed grey line), illustrating its sensitivity to this lens' position. With a customized design of the cavity parameters and cylindrical lens, the maxima of this curve can be tailored to match the waveguide mode size, thus providing a reasonable positional tolerance range $+/-0.2 \mathrm{~mm}$.

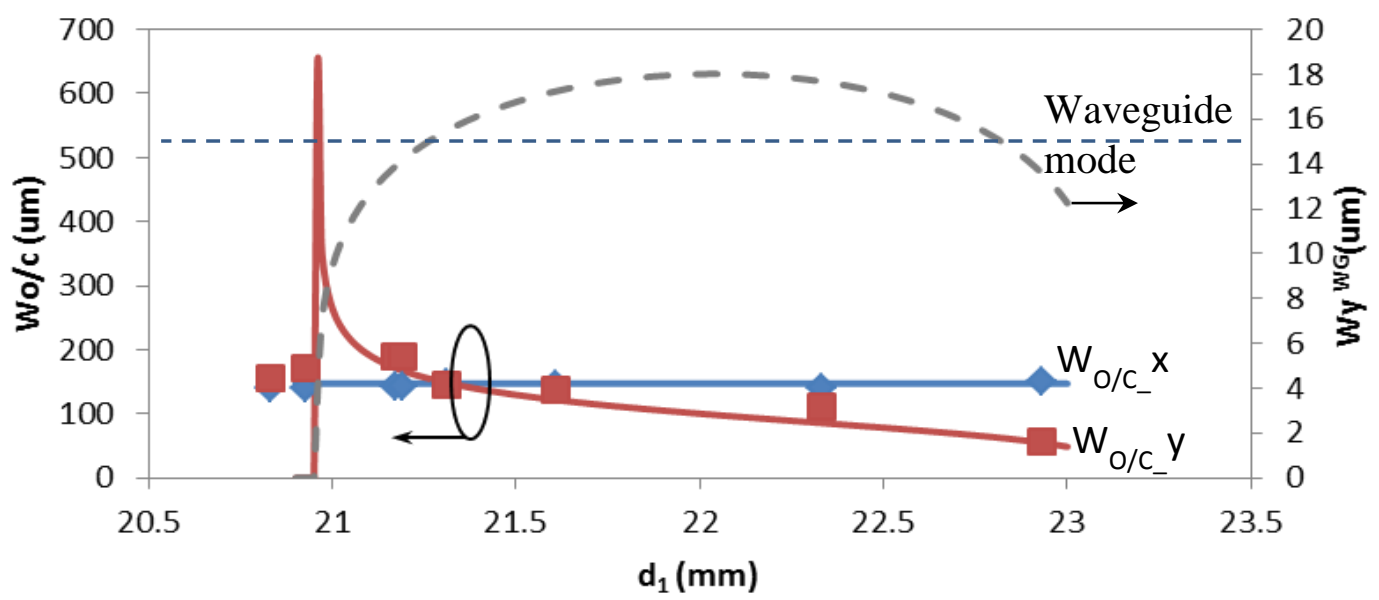

Figure 2: Beam radii at cavity out-coupling mirror as a function of $\mathrm{d}_{1}$ position in-the-plane (x) and waveguide (y) axes, obtained from experiment $(\bullet, \bullet)$ and simulation (lines).

Having set $d_{1}$ to a position for good coupling efficiency we incremented the pump power and recorded the near- and farfield beam information, Figure 3 a shows the dependence of the in-plane cavity beam waist radii on the respective pump power. We obtain the effective thermal lens at each power level by fitting the thermal-lens dioptric power versus pump power, Figure $3 \mathrm{~b}$, giving a linear dependence with a gradient of $0.024 \mathrm{~m}^{-1} \mathrm{~W}^{-1}$. Figure 4 illustrates the effective thermal lens that would produce the measured fundamental embedded-mode beam radii at the output-coupling mirror for our cavity configuration. The PW was found to have a thermally induced lens with a focal length of $\sim 2.5 \mathrm{~m}$ near the lasing threshold condition, which reduced to around $0.46 \mathrm{~m}$ at the full available incident pump power of $87 \mathrm{~W}$. Generally, the experimental data and fitted effective thermal lens showed very good agreement with the fitted hyperbolic dependence on pump power. Moreover as shown in Figure 4, the calculated fundamental cavity mode size, back-propagated from the 
measured beam waist at the output coupler to the PW, varied over a relatively small range for the pump powers investigated, i.e. $~ 70-80 \%$ of the in-plane pump-beam width averaged over the length of the PW.
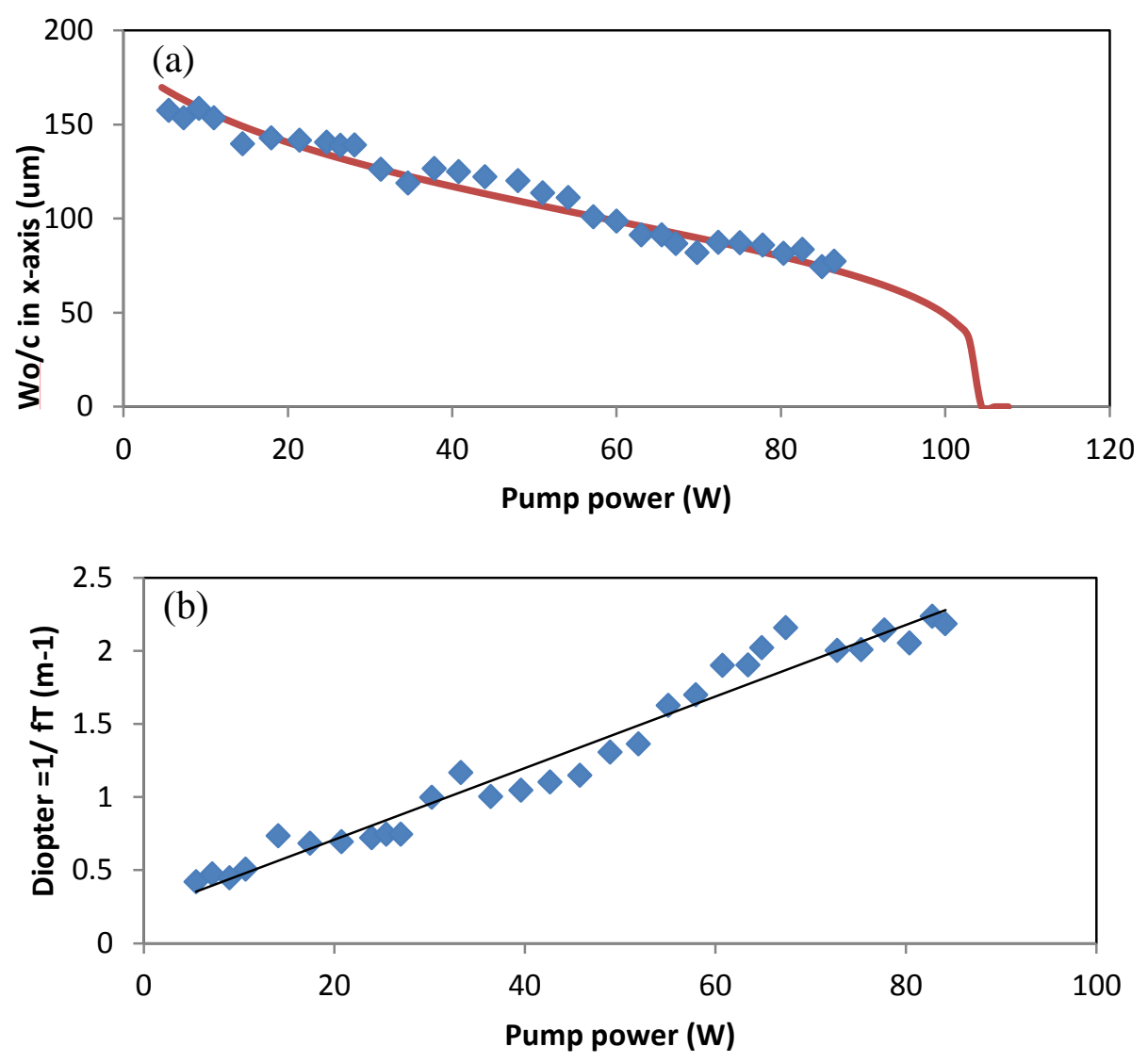

Figure 3: Dependence of the (a) in-plane cavity beam waist radii, and (b) diopter power of the effective thermal focal length on the respective pump power.

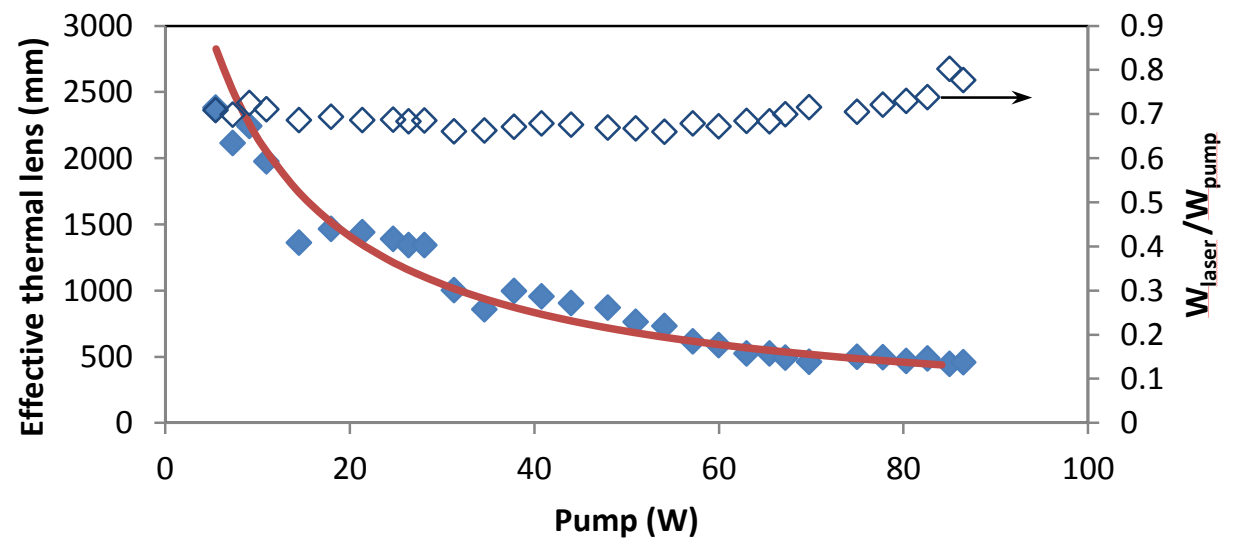

Figure 4: Effective thermal focal length deduced from the experimental results (diamonds), and fitted with hyperbolic expression (line) with respect to pump power. The right vertical axis of the plot gives the calculated fundamental cavity mode ratio to the average pump beam radius in PW. 
Utilizing our characterization setup we could monitor the beam characteristics while optimising the laser, looking for the smallest beam size in the far-field image (i.e. lowest divergence). In reality, this is only true if the fundamental mode beam waist at the output-coupling mirror is unchanged, hence the necessity to monitor both. Figure 5 shows the output power of the $946 \mathrm{~nm}$ PW laser together with $\mathrm{M}^{2}$ parameter at each of the measured power levels. Generally, the laser was operating on at least two transverse modes in-plane attributed to the slightly smaller cavity-to-pump mode overlap ratio as indicated in Figure 4. At the highest average output power of $29 \mathrm{~W} \mathrm{CW}$ the beam propagation parameters in the respective axes were $\mathrm{M}^{2}{ }_{\mathrm{x}}=3.2$ by $\mathrm{M}_{\mathrm{y}}^{2}=2.4$ giving a radiance of $4.3 \mathrm{TWm}^{-2} \mathrm{sr}^{-1}$. To the best of our knowledge this is the highest radiance reported for this $946 \mathrm{~nm}^{4} \mathrm{~F}_{3 / 2} \rightarrow{ }^{4} \mathrm{I}_{9 / 2}$ transition in Nd:YAG.

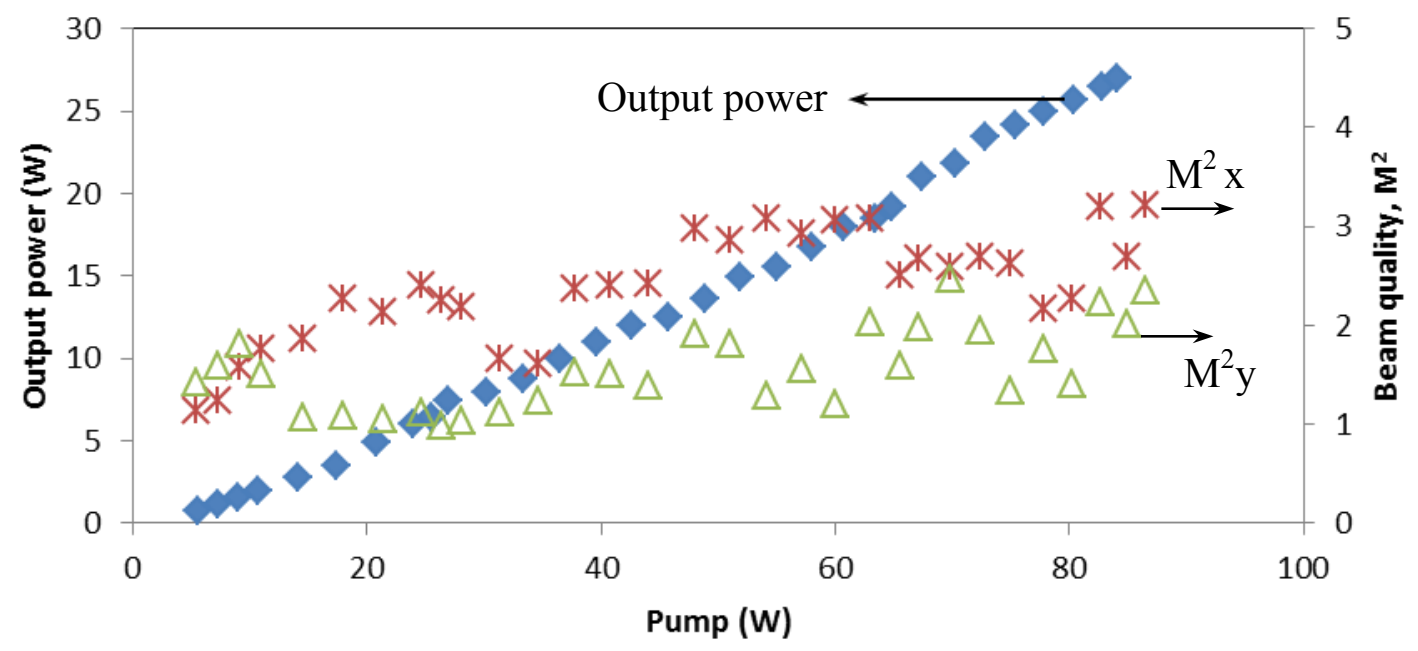

Figure 5: Laser power plot and output beam quality performance in guided- (triangle) and unguided-axis (asterisk).

\section{CONCLUSIONS}

In conclusion, we have determined the effective thermal lens in a Nd:YAG double-clad PWL using a near-field, far-field beam characterization technique that provided explicit simultaneous measurement of the laser's beam-parameter product, from which we could determine the mode size throughout the cavity dependent upon the pumping power. This technique is far superior to the conventional mechanical beam quality measurement methods in terms of providing a full characterization of the irradiance distribution, when using a CCD array, and effectively real-time measurement of the beam propagation parameter. Operating at a lasing wavelength of $946 \mathrm{~nm}$ and pumping powers up to $87 \mathrm{~W}$ the effective thermal lens in the plane of waveguide was measured to be $>0.45 \mathrm{~m}$. For the optimised laser system we obtained $29 \mathrm{~W}$ of average output power with radiance of $4.3 \mathrm{TWm}^{-2} \mathrm{sr}^{-1}$ representing, to our knowledge, the highest radiance output for this quasi-four-level $\mathrm{Nd}$ :YAG laser transition.

\section{REFERENCES}

[1] Fan, T. Y., Byer, R. L., "Modeling and cw operation of a quasi-three-level $946 \mathrm{~nm}$ Nd:YAG laser," IEEE J. Quantum Electron. QE-23, 605-612 (1987).

[2] Zhou, R., Li, E., Li, H., Wang, P., Yao, J., "Continous-wave, 15.2 W diode-end-pumped Nd:YAG laser operating at 946 nm" Opt. Lett. 31, 1869-1871 (2006).

[3] Gao, J., Speiser, J., Giesen, A., "25-W diode-pumped continuous-wave quasi-three-level Nd:YAG thin disk laser," in Technical Digest of Advanced Solid-State Photonics, Vienna, Austria, paper TuB34 (2005).

[4] Délen, X., Martial, I., Didierjean, J., Aubry, N., Sangla, D., Balembois, F., Georges, P., "34W continuous wave Nd:YAG single crystal fiber laser emitting at 946 nm,” Appl. Phys. B 104, 1-4 (2011).

[5] Mackenzie, J. I., Li, C., Shepherd, D., "Multi-watt, high efficiency, diffraction-limited Nd:YAG planar waveguide laser," J. Quantum Electron. QE-39, 493-500 (2003).

[6] Mackenzie, J. I., “An efficient high-power 946 nm Nd:YAG planar waveguide laser,” Appl. Phys. B 97, 297-306 (2009). 
[7] Mackenzie, J. I., "Power-scaling Nd:YAG's quasi-four-level transition,” Proc. of SPIE 7578, 75780L-1-8 (2010).

[8] Neuenschwander, B., Weber, R., Weber, H. P., "Determination of the thermal lens in solid-state lasers with stable cavities," J. Quantum Electron. QE-31, 1082-1087 (1995).

[9] DeSilvestri, S., Laporta, P., Mague, V., "Pump power stability range of single-mode solid-state lasers with rod thermal lensing," IEEE J. Quantum Electron. QE-23, 1999-2004 (1987).

[10] Innocenzi, M. E., Yura, H. T., Fincher, C. L., Fields, R. A., "Thermal modeling of continuous-wave end-pumped solid-state lasers," Appl. Phys. Lett. 56, 1831-1833 (1990).

[11] Song, F., Zhang, C. B., Ding, X., Xu, J. J., Zhang, G. Y., Leigh, M., Peyghambarian, N., "Determination of thermal focal length and pumping radius in gain medium in laser-diode-pumped Nd: $\mathrm{YVO}_{4}$ lasers," Appl. Phys. Lett. 81, 2145-2147 (2002).

[12] Blows, J., Dawes, J. M., Omatsu, T., "Thermal lensing measurements in line-focus end-pumped neodymium yttrium aluminum garnet using holographic lateral shearing interferometry," J. Appl. Phys. 83, 2901-2906 (1998).

[13] Tidwell, S. C., Seamans, J. F., Bowers, M. S., Cousins, A. K., "Scaling CW diode-end-pumped Nd:YAG lasers to high average powers," IEEE J. Quantum Electron. QE-28, 997-1009 (1992).

[14] Murray, J. E., "Pulsed gain and thermal lensing of Nd:LiYF 4 ," IEEE J.Quantum Electron. QE-19, 488-491 (1983).

[15] P. J. Hardman, W. A. Clarkson, G. J. Friel, M. Pollnau, and D. C. Hanna, "Energy-transfer upconversion and thermal lensing in high-power end-pumped Nd:YLF laser crystals," IEEE J. Quantum Electron. QE-35, 647-655 (1999).

[16] Hodgson, N., Weber, H., [Optical Resonators], Berlin, Springer (1996).

[17] Siegman, A. E., [Lasers], Mill Valley, CA, University Science Books (1986). 